\title{
Heat conduction equation in physically inhomogeneous moving composite solids
}

\author{
G. R. Gasimov ${ }^{1}$ and Z. A. Abusutash ${ }^{2 *}$ \\ ${ }^{1}$ Baku State University, Faculty of applied mathematics, Baku. \\ ${ }^{2}$ Department of Mathematics, Faculty of Science, Damietta University, Damietta 34517, Egypt. \\ * Permanent address: El-Mergeb University, Faculty of Science, Mathematics Department, Libya.
}

Received: 11 January 2016/Accepted: 2 April 2016

* Corresponding author: mooon_smile16@yahoo.com

\begin{abstract}
This paper presents a heat conduction problem of inhomogeneous physically moving compound bodies, which consists of two cylinders. Using a sequence of integral transformations(Laplace, Hankel), the Cauchys residue theory, Bessel functions and using the results of the roots of one transcendental equation, which results from the conditions of contact between the two cylinders, a solution in the form of a series is obtained. A special case of alternating zeros of transcendental equation is also given.
\end{abstract}

Keywords: A moving cylinder; Composite solids; Heat conduction problem.

\section{Introduction}

The problems of heat conduction in composite solids are usually solved by the sequence of integral transformations method. Thermal conductivity problem of the moving solid bodies which depends on two cylinders has been considered with by researchers among of them Carslaw and Jaeger [2].

A simple form of the thermal conductivity problem has been initiated almost a century ago, for many of simple moving and simple geometrical regions and all its earlier details have been summarized and given by Carslaw and Jaeger [2] and Özisik [3]. In recent years, a number of the papers investigated the problem of heat conduction in the circular a hollow cylinder using a Maple11 program; see Shahout et al.[4].Analytical solution of the problems thermal conductivity of the moving bodies with finite size (in continuous and hollow cylinders and in parallelepipeds) is obtained by Gasimov et al. [5], Lotarev [6] and Kuznetsova [7]. Kholodovskii [8] considered boundary value problems for linear differential equations in piecewise - homogenous cylinders into two half-cylinders by multilayer film and these problems have a great importance in many engineering fields which intervene in the design of internal combustion engines, material in aviation, and the factories of the production of military weapons. Our motivation in this paper is to investigate the problem of heat propagation in an inhomogeneous composite solid with variable thermic features and with composite region which consists of two cylinders, and moves with velocity $v$ in the direction of z-axis. We use sequential of integral transformations like Laplace and Hankel transformations. Our idea is to choose appropriate integral transformation with respect to each 
variable which allows overcoming the main difficulty to find the desired solution with the help of inverse transformations.

\section{Formulation of the problem}

Consider the composite solid which consists of two parts: a hollow cylinder $(a<\mathrm{r}<\mathrm{b}, 0<\mathrm{z}<$ $\left.\ell_{1}\right)$ and entire cylinder $\left(0<\mathrm{r}<\mathrm{b}, \ell_{1}<z<\right.$ $\ell_{1}+\ell_{2}$ ), which moves with velocity $v$ in the direction of the axis $o z$ where the heat flow is radial and cylindrically symmetric. At each parts, the initial temperature is given, the interior wall of the hollow cylinder is maintained at zero temperature. It is also sometimes referred to as 'Newton's Law', and from the external surface there happens radiation to the medium with zero temperature. On the joining surface (for $\mathrm{z}=\ell_{1}$ ) there is no contact resistance.

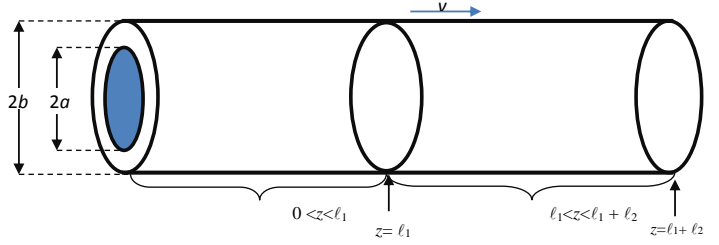

Fig. 1.

The formulation of this problem looks as follows:

$$
\begin{aligned}
& \frac{\partial \mathrm{T}_{1}}{\partial \mathrm{t}}=\varkappa_{1}\left(\frac{\partial^{2} \mathrm{~T}_{1}}{\partial \mathrm{r}^{2}}+\frac{1}{\mathrm{r}} \frac{\partial \mathrm{T}_{1}}{\partial \mathrm{r}}+\frac{\partial^{2} \mathrm{~T}_{1}}{\partial \mathrm{z}^{2}}\right)-\mathrm{v} \\
& \frac{\partial \mathrm{T}_{1}}{\partial \mathrm{z}}, \quad a<\mathrm{r}<\mathrm{b}, 0<\mathrm{z}<\ell_{1}, \mathrm{t}>0,
\end{aligned}
$$

$\left.\mathrm{T}_{1}\right|_{\mathrm{t}=0}=\mathrm{f}_{1}(\mathrm{r}, \mathrm{z})$,

(2)

$\left.\mathrm{T}_{1}\right|_{\mathrm{r}=a}=0$,

$\left.\left(\frac{\partial}{\partial \mathrm{r}}+\mathrm{h}_{1}\right) \mathrm{T}_{1}\right|_{\mathrm{r}=\mathrm{b}}$

$=0$,

$\left.\left(\frac{\partial}{\partial \mathrm{z}}-g_{1}\right) \mathrm{T}_{1}\right|_{\mathrm{z}=0}$

$=0$;

$\frac{\partial \mathrm{T}_{2}}{\partial \mathrm{t}}=\mathcal{H}_{2}\left(\frac{\partial^{2} \mathrm{~T}_{2}}{\partial \mathrm{r}^{2}}+\frac{1}{\mathrm{r}} \frac{\partial \mathrm{T}_{2}}{\partial \mathrm{r}}+\frac{\partial^{2} \mathrm{~T}_{2}}{\partial \mathrm{z}^{2}}\right)-v$

$\frac{\partial \mathrm{T}_{2}}{\partial \mathrm{z}} \quad, \quad 0<\mathrm{r}<\mathrm{b}, \ell_{1}<\mathrm{z}<\ell_{1}+\ell_{2} \quad, \mathrm{t}>0$

$\left.\mathrm{T}_{2}\right|_{\mathrm{t}=0}=\mathrm{f}_{2}(\mathrm{r}, \mathrm{z})$,

(6)

$$
\begin{aligned}
& \left.\left(\frac{\partial}{\partial \mathrm{r}}+\mathrm{h}_{2}\right) \mathrm{T}_{2}\right|_{\mathrm{r}=\mathrm{b}} \\
& =0 \\
& \left.\left(\frac{\partial}{\partial \mathrm{z}}+g_{2}\right) \mathrm{T}_{2}\right|_{\mathrm{z}=\ell_{1}+\ell_{2}}=0, \quad 0<\mathrm{r} \\
& <\mathrm{b}, \\
& \left.\mathrm{T}_{2}\right|_{\mathrm{z}=\ell_{1}}=0, \quad 0 \leq \mathrm{r} \\
& <a .
\end{aligned}
$$

In the first part, if we assume that there is no contact resistance at the surface of separation $\mathrm{z}=\ell_{1}$, the boundary conditions become

$$
\begin{aligned}
& \left.\mathrm{T}_{1}\right|_{\mathrm{z}=\ell_{1}}=\left.\mathrm{T}_{2}\right|_{\mathrm{z}=\ell_{1}}{ }^{\prime} \\
& \left.\mathrm{k}_{1} \frac{\partial \mathrm{T}_{1}}{\partial \mathrm{z}}\right|_{\mathrm{z}=\ell_{1}}=\left.\mathrm{k}_{2} \frac{\partial \mathrm{T}_{2}}{\partial \mathrm{z}}\right|_{\mathrm{z}=\ell_{1}}, a<r \\
& <\mathrm{b},
\end{aligned}
$$

Where: $a$ and $b$ are the radii of the hollow cylinder and the entire cylinder, respectively. In the first region

$0<\mathrm{z}<\ell_{1} . \quad \mathrm{T}_{1}, \rho_{1,}, \mathrm{c}_{1}$ and $\mathcal{H}_{1}$ are the temperature, density, specific heat and diffusivity, whereas $T_{2}, \rho_{2}, c_{2}$ and $\varkappa_{2}$ for the corresponding quantities in $\ell_{1}<\mathrm{z}<\ell_{1}+$ $\ell_{2} \quad \mathrm{k}_{1}$ and $\mathrm{k}_{2}$ are the thermal conductivities of

substance, that move with velocity $v$ is constant, and $\mathrm{h}_{1}, \mathrm{~h}_{2}, g_{1}$ and $g_{2}$ are the coefficients of surface heat transfer which are numbed to be constants, and $\mathrm{f}_{1}(\mathrm{r}, \mathrm{z})$ and $\mathrm{f}_{2}(\mathrm{r}, \mathrm{z})$ are given functions .

\section{Solving Method}

Using successive transformations, the solution is organized follows.

The first stage: We apply the Laplace transform with respect to $\mathrm{t}$, and Hankel transform in $a<$ $\mathrm{r}<\mathrm{b}$. The problem is transformed into

$$
\begin{aligned}
& \mathcal{H}_{1}\left(-\lambda^{2} \overline{\widetilde{\mathrm{T}}}_{1}+\frac{\mathrm{d}^{2} \overline{\widetilde{\mathrm{T}}}_{1}}{\mathrm{dz}^{2}}\right)-v \frac{\mathrm{d} \overline{\widetilde{\mathrm{T}}}_{1}}{\mathrm{dz}}-\mathrm{p} \overline{\widetilde{\mathrm{T}}}_{1} \\
& =-\overline{\mathrm{f}}_{1}(\mathrm{z}), 0<\mathrm{z} \\
& <\ell_{1}, \\
& \left.\left(\frac{\mathrm{d}}{\mathrm{dz}}-g_{1}\right) \overline{\widetilde{\mathrm{T}}}_{1}\right|_{\mathrm{z}=0}=0,
\end{aligned}
$$

We write $\widetilde{T}_{i}$ for the Laplace transform of $T_{i}$ with respect to $t$, and $\overline{\widetilde{T}}_{1}$ for the Hankel transform of $\widetilde{\mathrm{T}}_{1}$ with respect to $\mathrm{r}$, respectively; 


$$
\begin{aligned}
& \widetilde{\mathrm{T}}_{\mathrm{i}} \equiv \widetilde{\mathrm{T}}_{\mathrm{i}}(\mathrm{r}, \mathrm{z} ; \mathrm{p})=\int_{0}^{\infty} \mathrm{e}^{-\mathrm{pt}} \mathrm{T}_{i}(\mathrm{r}, \mathrm{z}, \mathrm{t}) \mathrm{dt}, \\
& (\operatorname{Re}(\mathrm{p})>0), \quad \mathrm{i}=1,2 . \\
& \overline{\widetilde{\mathrm{T}}}_{1} \equiv \overline{\widetilde{\mathrm{T}}}_{1}(\mathrm{z} ; \mathrm{p}, \eta)=\int_{a}^{b} \mathrm{r} \widetilde{\mathrm{T}}_{1}(\mathrm{r}, \mathrm{z} ; \mathrm{p}) \overline{\mathrm{K}}_{\eta}^{(1)}(\mathrm{r}) \mathrm{dr} \text {. } \\
& \left(\overline{\mathrm{f}}_{1}(\mathrm{z})=\int_{a}^{b} \mathrm{rf}_{1}(\mathrm{r}, \mathrm{z}) \overline{\mathrm{K}}_{\eta}^{(1)}(\mathrm{r}) \mathrm{dr}\right) .
\end{aligned}
$$

$\overline{\mathrm{K}}_{\eta}^{(1)}(\mathrm{r})$ is considered the solution of the spectral problem of Bessel's equation;

$\frac{\mathrm{d}^{2} \overline{\mathrm{K}}^{(1)}}{\mathrm{dr}^{2}}+\frac{1}{\mathrm{r}} \frac{\mathrm{d} \overline{\mathrm{K}}^{(1)}}{\mathrm{dr}}+\lambda^{2} \overline{\mathrm{K}}^{(1)}=0$,

$a<\mathrm{r}<\mathrm{b}$

$$
\left.\overline{\mathrm{K}}^{(1)}\right|_{\mathrm{r}=a}=0,\left.\quad\left(\frac{\mathrm{d} \overline{\mathrm{K}}^{(1)}}{\mathrm{dr}}+\mathrm{h}_{1} \overline{\mathrm{K}}^{(1)}\right)\right|_{\mathrm{r}=\mathrm{b}}=
$$

0 .

The general solution of (13) is:

$\overline{\mathrm{K}}^{(1)}(\mathrm{r})=\mathrm{A}_{0} \mathrm{~J}_{0}(\lambda \mathrm{r})+\mathrm{B}_{0} \mathrm{Y}_{0}(\lambda \mathrm{r})$;

where $\mathrm{J}_{0}, \mathrm{Y}_{0}$ are Bessel functions of zero order $\mathrm{A}_{0}$ and $\mathrm{B}_{0}$ are arbitrary constants.

The unknowns $A_{0}$ and $B_{0}$ are to be found from (14), as follows:

$$
\mathrm{A}_{0} \mathrm{~J}_{0}(\lambda a)+\mathrm{B}_{0} \mathrm{Y}_{0}(\lambda a)=0 \text {, }
$$

$$
\begin{gathered}
\mathrm{A}_{0}\left[\lambda \mathrm{J}_{0}^{\prime}(\lambda \mathrm{b})+\mathrm{h}_{1} \mathrm{~J}_{0}(\lambda \mathrm{b})\right] \\
+\mathrm{B}_{0}\left[\lambda \mathrm{Y}_{0}^{\prime}(\lambda \mathrm{b})+\mathrm{h}_{1} \mathrm{Y}_{0}(\lambda \mathrm{b})\right]=0 .
\end{gathered}
$$

So, the eigenvalues of the spectral problem (13) and (14) are obtained from the determinant system (15):

$$
\left|\begin{array}{cc}
\mathrm{J}_{0}(\lambda a) & \mathrm{Y}_{0}(\lambda a) \\
\lambda \mathrm{J}_{0}^{\prime}(\lambda \mathrm{b})+\mathrm{h}_{1} \mathrm{~J}_{0}(\lambda \mathrm{b}) & \lambda \mathrm{Y}_{0}^{\prime}(\lambda \mathrm{b})+\mathrm{h}_{1} \mathrm{Y}_{0}(\lambda \mathrm{b})
\end{array}\right|
$$

or,

$$
\begin{aligned}
& \mathrm{h}_{1}\left[\mathrm{~J}_{0}(\lambda a) \mathrm{Y}_{0}(\lambda b)-\mathrm{J}_{0}(\lambda b) \mathrm{Y}_{0}(\lambda a)\right]+ \\
& \lambda\left[\mathrm{J}_{1}(\lambda b) \mathrm{Y}_{0}(\lambda a)-\mathrm{J}_{0}(\lambda a) \mathrm{Y}_{1}(\lambda b)\right]=0 . \\
& (16)
\end{aligned}
$$

The roots of (16) are all real and simple; we need to prove the following theorem:

Theorem (Type theorem Dixon's [1]):

If $\mathrm{AD}=\mathrm{BC}$, then the positive zeros of $\mathrm{AY}_{0}(\mathrm{x})+$ $\mathrm{BxY}_{0}^{\prime}(\mathrm{x})$ are interlaced with those of $\mathrm{CJ}_{0}(\mathrm{x})+$ $\operatorname{DxJ}_{0}^{\prime}(\mathrm{x})$.

To prove this theorem, we enter a function in the following form

$$
\varphi(\mathrm{x})=\frac{\mathrm{CJ}_{0}(\mathrm{x})+\mathrm{DxJ}_{0}^{\prime}(\mathrm{x})}{\mathrm{AY}_{0}(\mathrm{x})+\mathrm{BxY}_{0}^{\prime}(\mathrm{x})},
$$

Let us denote $\mathrm{d} \equiv\left[\mathrm{AY}_{0}(\mathrm{x})+\mathrm{BxY}_{0}^{\prime}(\mathrm{x})\right]^{2}$; and we need to prove that this is an increasing function or is a decreasing function between any two consecutive zeros of the denominator, then

$$
\begin{aligned}
& \varphi^{\prime}(\mathrm{x})=\frac{1}{\mathrm{~d}}\left\{\left[(\mathrm{C}+\mathrm{D}) \mathrm{J}_{0}^{\prime}(\mathrm{x})+\mathrm{Dx}\right]_{0}^{\prime \prime}(\mathrm{x})\right]\left[\mathrm{AY}_{0}(\mathrm{x})\right. \\
& \left.+\mathrm{BxY}_{0}^{\prime}(\mathrm{x})\right] \\
& -\left[\mathrm{CJ}_{0}(\mathrm{x})+\mathrm{DxJ}_{0}^{\prime}(\mathrm{x})\right] \text {. } \\
& \text {. } \left.\left[(\mathrm{A}+\mathrm{B}) \mathrm{Y}_{0}^{\prime}(\mathrm{x})+\mathrm{BxY}_{0}^{\prime \prime}(\mathrm{x})\right]\right\}=\frac{1}{\mathrm{~d}}\{[(\mathrm{C}+ \\
& \text { D) } \left.J_{0}^{\prime}(\mathrm{x})-\mathrm{D} \mathrm{J}_{0}^{\prime}(\mathrm{x})-\mathrm{Dx} \mathrm{J}_{0}(\mathrm{x})\right]\left[\mathrm{AY}_{0}(\mathrm{x})+\right. \\
& \left.\mathrm{BxY}_{0}^{\prime}(\mathrm{x})\right]- \\
& -\left[\mathrm{CJ}_{0}(\mathrm{x})+\mathrm{DxJ}_{0}^{\prime}(\mathrm{x})\right]\left[(\mathrm{A}+\mathrm{B}) \mathrm{Y}_{0}^{\prime}(\mathrm{x})-\mathrm{BY}_{0}^{\prime}(\mathrm{x})\right. \\
& \left.\left.-\mathrm{BxY}_{0}(\mathrm{x})\right]\right\}= \\
& =\frac{1}{\mathrm{dx}}\left\{\frac{2}{\pi}\left[\mathrm{AC}+\mathrm{BDx}^{2}\right]+[\mathrm{BC}-\mathrm{AD}]\left[\mathrm{x}^{2} \mathrm{~J}_{0}(\mathrm{x}) \mathrm{Y}_{0}(\mathrm{x})\right.\right. \\
& \left.\left.+\mathrm{J}_{0}^{\prime}(\mathrm{x}) \mathrm{Y}_{0}^{\prime}(\mathrm{x})\right]\right\} \text {. } \\
& \mathrm{BC}-\mathrm{AD}=0 \Rightarrow \exists \mathrm{S}: \frac{\mathrm{A}}{\mathrm{C}}=\frac{\mathrm{B}}{\mathrm{D}} \equiv \mathrm{S} \Rightarrow \mathrm{A} \\
& =\text { C.S }, \mathrm{B}=\mathrm{D} . \mathrm{S} \Rightarrow
\end{aligned}
$$

if $S>0$, then $A C=C^{2} . S>0$ and $B D=$ $\mathrm{D}^{2} . \mathrm{S}>0$, if $\mathrm{S}<0$, then $\mathrm{AC}<0, \mathrm{BD}<0$.

So form the sign of $\varphi^{\prime}(\mathrm{x})$, fixed between any two zeros consecutive for the denominator.

Again, we next use this theorem to rewrite (16) as $\frac{\mathrm{J}_{0}(\beta \mathrm{x})}{\mathrm{Y}_{0}(\beta \mathrm{x})}=\frac{\mathrm{h}_{1} \mathrm{bJ}_{0}(\mathrm{x})+\mathrm{xJ}_{0}^{\prime}(\mathrm{x})}{\mathrm{h}_{1} \mathrm{bY}_{0}(\mathrm{x})+\mathrm{xY}_{0}^{\prime}(\mathrm{x})}$,

Where $\beta \equiv \frac{a}{b} \quad, \quad \mathrm{x} \equiv \lambda \mathrm{b}$.

Using this theorem for the right hand side of (17) at $\mathrm{A}=\mathrm{C}=\mathrm{h}_{1} \mathrm{~b}, \mathrm{~B}=\mathrm{D}=1$, and using the properties of Bessel function $\mathrm{J}_{0}, \mathrm{Y}_{0}$ [1], all roots of equation (16) are real, simple and have infinite numbers.

Now in the special case for (16), we made our calculations, using program Maple and part of this programming, supposing that the thickness of the cylinder is $10^{-3} \mathrm{~m}^{2}$, and considering the center material and the cylinder material are silver $\left(\mathrm{k}_{1}=\right.$ $\left.1.00, \mathrm{~h}_{1}=0.07\right)$. Calculating the roots of the equation (16) rewriting in Maple language, as well as taking the values to $a=1, \mathrm{~b}=2$, we obtain the following [4] by:

restart;

$$
\begin{aligned}
\Phi:= & \text { unapply }\left(\lambda \cdot \left(\operatorname{BesselJ}(0, \lambda) \cdot \frac{\partial}{\partial \lambda}(\operatorname{BesselY}(0,2 \cdot \lambda))\right.\right. \\
& \left.-\operatorname{BesselY}(0, \lambda) \cdot \frac{\partial}{\partial \lambda}(\operatorname{BesselJ}(0,2 \cdot \lambda))\right)+0.07 \cdot(\operatorname{BesselJ}(0 \\
& \lambda) \cdot \operatorname{BesselY}(0,2 \cdot \lambda)-\operatorname{BesselY}(0, \lambda) \cdot \operatorname{BesselJ}(0,2 \cdot \lambda))): \Phi(0)
\end{aligned}
$$


$\lambda(-2 \operatorname{BesselJ}(0, \lambda) \operatorname{BesselY}(1,2 \lambda)+2 \operatorname{BesselY}(0, \lambda) \operatorname{BesselJ}(1$, $2 \lambda))+0.07 \operatorname{BesselJ}(0, \lambda) \operatorname{BesselY}(0,2 \lambda)-0.07 \operatorname{BesselY}(0$, $\lambda) \operatorname{BesselJ}(0,2 \lambda)$

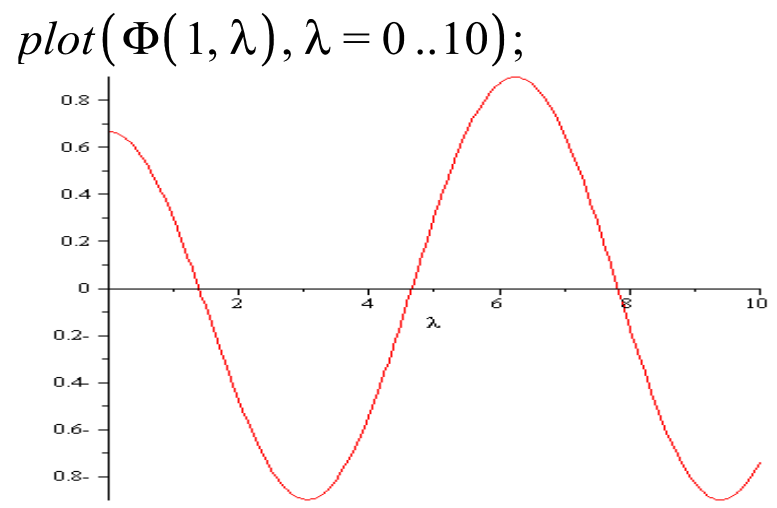

Fig (2)

lambda $[1,1]:=$ fsolve $(\Phi(1), \lambda, 1 . .2)$ 1.388691949

for $k$ from 2 to 10 do lambda $[1, k]:=$ ssolve $(\Phi(1), \lambda, \%+\pi . . \%+2$ $\pi)$ od

$4.653498141,7.818655693$,

10.97034008

$14.11753953,17.26270039$,

$20.40676348, \quad 23.55016784, \quad 26.69314596$, 29.83583241

The two solutions of the equations can be found as:

$$
\begin{aligned}
& A_{0}=\lambda_{1, \eta} Y_{0}^{\prime}\left(\lambda_{1, \eta} b\right)+h_{1} Y_{0}\left(\lambda_{1, \eta} b\right), \\
& B_{0}=-\lambda_{1, \eta} J_{0}^{\prime}\left(\lambda_{1, \eta} b\right)-h_{1} J_{0}\left(\lambda_{1, \eta} b\right) .
\end{aligned}
$$

Therefore one can consider the following functions as eigen functions for problems (13)(14):

$$
\begin{gathered}
\Phi_{1, \eta}(r) \\
=\left[\lambda_{1, \eta} \mathrm{Y}_{0}^{\prime}\left(\lambda_{1, \eta} \mathrm{b}\right)+\mathrm{h}_{1} \mathrm{Y}_{0}\left(\lambda_{1, \eta} \mathrm{b}\right)\right] \mathrm{J}_{0}\left(\lambda_{1, \eta} \mathrm{r}\right) \\
-\left[\lambda_{1, \eta} \mathrm{J}_{0}^{\prime}\left(\lambda_{1, \eta} \mathrm{b}\right)+\mathrm{h}_{1} \mathrm{~J}_{0}\left(\lambda_{1, \eta} \mathrm{b}\right)\right] \mathrm{Y}_{0}\left(\lambda_{1, \eta} \mathrm{r}\right),
\end{gathered}
$$
and the kernel can be found from a relation:$$
\overline{\mathrm{K}}_{\eta}^{(1)}(\mathrm{r})=\frac{1}{\mathrm{~N}_{1, \eta}} \Phi_{1, \eta}(\mathrm{r}) \text {, }
$$

where

$$
\mathrm{N}_{1, \eta}=\int_{a}^{\mathrm{b}} \mathrm{r}\left[\Phi_{1, \eta}(\mathrm{r})\right]^{2} \mathrm{dr} .
$$

Thus, our solution for (19)-(20) is in the form $\overline{\widetilde{T}}_{1, \eta}(z ; p, \eta)$

$=e^{v z /\left(2 \varkappa_{1}\right)}\left[\frac{2}{\omega_{1}-\mathrm{q}_{1, \eta}}\left(\omega_{1} \sinh \mathrm{q}_{1, \eta} \mathrm{z}\right.\right.$

$\left.-q_{1, \eta} \cosh q_{1, \eta} z\right) A_{1, \eta}$

$$
\begin{aligned}
& -\frac{1}{\mathcal{\varkappa}_{1} \mathrm{q}_{1, \eta}} \int_{0}^{\mathrm{z}} \mathrm{e}^{-v \zeta /\left(2 \varkappa_{1}\right)} \sinh \mathrm{q}_{1, \eta}(\mathrm{z} \\
& \left.-\zeta) \cdot \overline{\mathrm{f}}_{1, \eta}(\zeta) \mathrm{d} \zeta\right]
\end{aligned}
$$

where

$$
\mathrm{q}_{1, \eta}^{2} \equiv\left[\mathrm{p}+\left(\varkappa_{1} \lambda_{1, \eta}^{2}+\frac{v^{2}}{4 \varkappa_{1}}\right)\right] / \varkappa_{1}, \omega_{1} \equiv \frac{v}{2 \varkappa_{1}}-
$$

$g_{1}, \mathrm{~A}_{1, \eta}$ are arbitrary constants .

The second stage: Applying a sequence of the Laplace transformation and the zero-order Hankel transform with respect to $r$ to (5)-(8), gives

$$
\begin{aligned}
& \mathcal{H}_{2}\left(-\lambda^{2} \overline{\widetilde{\mathrm{T}}}_{2}+\frac{\mathrm{d}^{2} \overline{\widetilde{\mathrm{T}}}_{2}}{\mathrm{dz}^{2}}\right)-v \frac{\mathrm{d} \overline{\widetilde{\mathrm{T}}}_{2}}{\mathrm{dz}}-\mathrm{p} \overline{\widetilde{\mathrm{T}}}_{2} \\
& =-\overline{\mathrm{f}}_{2}(\mathrm{z}), \quad \ell_{1}<z \\
& <\ell_{1}+\ell_{2},
\end{aligned}
$$

$\left.\left(\frac{\mathrm{d}}{\mathrm{dz}}+g_{2}\right) \overline{\widetilde{\mathrm{T}}}_{2}\right|_{\mathrm{z}=\ell_{1}+\ell_{2}}$

$=0$,

where $\overline{\widetilde{T}}_{2}$ is the Hankel transform, by kernel $\overline{\mathrm{K}}_{\eta}^{(2)}(\mathrm{r})$, for $\widetilde{\mathrm{T}}_{2}$ namely:

$$
\begin{gathered}
\overline{\widetilde{\mathrm{T}}}_{2} \equiv \overline{\widetilde{\mathrm{T}}}_{2}(\mathrm{z} ; \mathrm{p}, \eta)=\int_{0}^{\mathrm{b}} \mathrm{r} \widetilde{\mathrm{T}}_{2}(\mathrm{r}, \mathrm{z} ; \mathrm{p}) \overline{\mathrm{K}}_{\eta}^{(2)}(\mathrm{r}) \mathrm{dr} . \\
\left(\overline{\mathrm{f}}_{2}(\mathrm{z})=\int_{0}^{\mathrm{b}} \mathrm{rf}_{2}(\mathrm{r}, \mathrm{z}) \overline{\mathrm{K}}_{\eta}^{(2)}(\mathrm{r}) \mathrm{dr}\right) .
\end{gathered}
$$

$\overline{\mathrm{K}}_{\eta}^{(2)}(\mathrm{r})$ is considered as a solution of the spectral problem:

$\frac{\mathrm{d}^{2} \overline{\mathrm{K}}^{(2)}}{\mathrm{dr}^{2}}+\frac{1}{\mathrm{r}} \frac{\mathrm{d} \overline{\mathrm{K}}^{(2)}}{\mathrm{dr}}+\lambda^{2} \overline{\mathrm{K}}^{(2)}=0, \quad 0<r$

$<b$,

$$
\begin{aligned}
& \left.\overline{\mathrm{K}}^{(2)}\right|_{\mathrm{r}=0}<\infty, \quad\left(\frac{\mathrm{d} \overline{\mathrm{K}}^{(2)}}{\mathrm{dr}}+\mathrm{h}_{2}\right. \\
& \left.\overline{\mathrm{K}}^{(2)}\right)\left.\right|_{\mathrm{r}=\mathrm{b}}=0 .
\end{aligned}
$$

The eigenvalues $\lambda_{2, \eta}^{2}$ for the problems (20)-(21), can be obtained from the equation:

$\lambda \mathrm{J}_{0}^{\prime}(\lambda \mathrm{b})+\mathrm{h}_{2} \mathrm{~J}_{0}(\lambda \mathrm{b})=0$.

It is known that at [1], the roots of (22) are all real, simple and have infinite numbers.

The kernel $\overline{\mathrm{K}}_{\eta}^{(2)}(\mathrm{r})$ is calculated from the relation: $\overline{\mathrm{K}}_{\eta}^{(2)}(\mathrm{r})=\frac{1}{\mathrm{~N}_{2, \eta}} \mathrm{J}_{0}\left(\lambda_{2, \eta} \mathrm{r}\right)$,

where

$$
\begin{gathered}
\mathrm{N}_{2, \eta}=\int_{0}^{\mathrm{b}} \mathrm{rJ} \mathrm{J}_{0}^{2}\left(\lambda_{2, \eta} \mathrm{r}\right) \mathrm{dr}=\frac{\mathrm{b}^{2}}{2 \lambda_{2, \eta}^{2}}\left(\mathrm{~h}_{2}^{2}\right. \\
\left.+\lambda_{2, \eta}^{2}\right) \mathrm{J}_{0}^{2}\left(\lambda_{2, \eta} \mathrm{b}\right) .
\end{gathered}
$$


Thus, the solution of the problem (18)-(19) takes the form:

$\overline{\widetilde{\mathrm{T}}}_{2, \eta}(\mathrm{z} ; \mathrm{p}, \eta)$

$=e^{v \mathrm{z} /\left(2 \mathcal{\varkappa}_{2}\right)}\left\{\frac{2 \mathrm{e}^{\left(\ell_{1}+\ell_{2}\right) \mathrm{q}_{2, \eta}}}{\omega_{2}-\mathrm{q}_{2, \eta}}\left[\omega_{2} \sinh \mathrm{q}_{2, \eta}\left(\mathrm{z}-\left(\ell_{1}\right.\right.\right.\right.$

$\left.\left.\left.+\ell_{2}\right)\right)-\mathrm{q}_{2, \eta} \cosh \mathrm{q}_{2, \eta}\left(\mathrm{z}-\left(\ell_{1}+\ell_{2}\right)\right)\right] \cdot \mathrm{A}_{2, \eta}$

$+\frac{1}{\varkappa_{2} \mathrm{q}_{2, \eta}} \int_{\mathrm{z}}^{\ell_{1}+\ell_{2}} \mathrm{e}^{-v \zeta /\left(2 \varkappa_{2}\right)} \sinh \mathrm{q}_{2, \eta}(\mathrm{z}$

$\left.-\zeta) \cdot \overline{\mathrm{f}}_{2, \eta}(\zeta) \mathrm{d} \zeta\right\}$

where

$\mathrm{q}_{2, \eta}^{2} \equiv\left[\mathrm{p}+\left(\varkappa_{2} \lambda_{2, \eta}^{2}+\frac{v^{2}}{4 \varkappa_{2}}\right)\right] / \mathcal{\varkappa}_{2}, \omega_{2} \equiv \frac{v}{2 \varkappa_{2}}+$ $g_{2}, \mathrm{~A}_{2, \eta}$ are arbitrary constants .

The third stage: Using the inverse Hankel transform, we find two solutions of the two problems (11)-(12) and (18)-(19) in the form:

$\widetilde{\mathrm{T}}_{1}(\mathrm{r}, \mathrm{z} ; \mathrm{p})=2 \mathrm{e}^{\mathrm{vz} /\left(2 \varkappa_{1}\right)} \sum_{\eta=1}^{\infty} \frac{\mathrm{A}_{1, \eta}}{\omega_{1}-\mathrm{q}_{1, \eta}}\left(\omega_{1} \sinh \mathrm{q}_{1, \eta} \mathrm{z}\right.$ $-\frac{1}{\mathcal{x}_{1}} \sum_{\eta=1}^{\infty}\left[\int_{0}^{\mathrm{z}} \mathrm{e}^{v(\mathrm{z}-\zeta) /\left(2 \varkappa_{1}\right)} \sinh \mathrm{q}_{1, \eta}(\mathrm{z}\right.$

$\left.-\zeta) . \overline{\mathrm{f}}_{1, \eta}(\zeta) \mathrm{d} \zeta\right] \frac{1}{\mathrm{q}_{1, \eta}} \Phi_{1, \eta}(\mathrm{r})$,

$\widetilde{\mathrm{T}}_{2}(\mathrm{r}, \mathrm{z} ; \mathrm{p})$

$=2 \mathrm{e}^{v \mathrm{z} /\left(2 \mathcal{x}_{2}\right)} \sum_{\eta=1}^{\infty} \frac{\mathrm{A}_{2, \eta}}{\omega_{2}-\mathrm{q}_{2, \eta}}$

$\mathrm{e}^{\left(\ell_{1}+\ell_{2}\right) \mathrm{q}_{2, \eta}}\left[\omega_{2} \sinh \mathrm{q}_{2, \eta}\left(\mathrm{z}-\left(\ell_{1}+\ell_{2}\right)\right)-\mathrm{q}_{2, \eta}\right.$. . $\left.\cosh \mathrm{q}_{2, \eta}\left(\mathrm{z}-\left(\ell_{1}+\ell_{2}\right)\right)\right] \Phi_{2, \eta}(\mathrm{r})$

$+\frac{1}{\mathcal{H}_{2}} \sum_{\eta=1}^{\infty}\left[\int_{\mathrm{z}}^{\ell_{1}+\ell_{2}} \mathrm{e}^{v(\mathrm{z}-\zeta) /\left(2 \mathcal{\varkappa}_{2}\right)} \sinh \mathrm{q}_{2, \eta}(\mathrm{z}\right.$

$\left.-\zeta) . \overline{\mathrm{f}}_{2, \eta}(\zeta) \mathrm{d} \zeta\right] \frac{\Phi_{2, \eta}(\mathrm{r})}{\mathrm{q}_{2, \eta}}$,

where $\Phi_{2, \eta}(\mathrm{r})=J_{0}\left(\lambda_{2, \eta} \mathrm{r}\right)$.

When $a<r<\mathrm{b}$, the constants $\mathrm{A}_{1, \eta}$ and $\mathrm{A}_{2, \eta}$ are found from

$$
\begin{aligned}
& \left.\widetilde{\mathrm{T}}_{1}\right|_{\mathrm{z}=\ell_{1}}=\left.\widetilde{\mathrm{T}}_{2}\right|_{\mathrm{z}=\ell_{1}}{ }^{\prime} \\
& \left.\mathrm{k}_{1} \frac{\partial \widetilde{\mathrm{T}}_{1}}{\partial \mathrm{z}}\right|_{\mathrm{z}=\ell_{1}}=\left.\mathrm{k}_{2} \frac{\partial \widetilde{\mathrm{T}}_{2}}{\partial \mathrm{z}}\right|_{\mathrm{z}=\ell_{1}}, a<r \\
& <\mathrm{b},
\end{aligned}
$$

in the following forms

$A_{1, \eta}=\left(C_{2,2} \cdot G_{1, \eta}-C_{1,2} \cdot G_{2, \eta}\right) /\left(\Delta_{\eta} \cdot \Phi_{1, \eta}\right)$,

(26)

$A_{2, \eta}=\left(C_{1,1} \cdot G_{2, \eta}-C_{2,1} \cdot G_{1, \eta}\right) /\left(\Delta_{\eta} \cdot \Phi_{2, \eta}\right)$,

(27)

where
$\mathrm{C}_{1,1} \equiv \mathrm{C}_{1,1}(\eta)=\frac{2}{\omega_{1}-\mathrm{q}_{1, \eta}}\left(\omega_{1} \sinh \mathrm{q}_{1, \eta} \ell_{1}\right.$

$\mathrm{C}_{1,2} \equiv \mathrm{C}_{1,2}(\eta)$ $\left.-\mathrm{q}_{1, \eta} \cosh \mathrm{q}_{1, \eta} \ell_{1}\right) \cdot \mathrm{e}^{v \ell_{1} /\left(2 \varkappa_{1}\right)}$,

$=\frac{2}{\omega_{2}-q_{2, \eta}}\left(\omega_{2} \sinh q_{2, \eta} \ell_{2}\right.$

$\left.+\mathrm{q}_{2, \eta} \cosh \mathrm{q}_{2, \eta} \ell_{2}\right) \cdot \mathrm{e}^{\nu \ell_{1} /\left(2 \varkappa_{2}\right)} \cdot \mathrm{e}^{\left(\ell_{1}+\ell_{2}\right) \mathrm{q}_{2, \eta}}$,

$\mathrm{C}_{2,1} \equiv \mathrm{C}_{2,1}(\eta)=\frac{1}{\omega_{1}-\mathrm{q}_{1, \eta}}\left[\left(\frac{v \omega_{1}}{\varkappa_{1}}\right.\right.$

$\left.-2 \mathrm{q}_{1, \eta}^{2}\right) \sinh \mathrm{q}_{1, \eta} \ell_{1}$

$+\mathrm{q}_{1, \eta}\left(2 \omega_{1}\right.$

$\left.\left.-\frac{v}{\varkappa_{1}}\right) \cosh \mathrm{q}_{1, \eta} \ell_{1}\right] \mathrm{e}^{v \ell_{1} /\left(2 \varkappa_{1}\right)}$,

$\mathrm{C}_{2,2} \equiv \mathrm{C}_{2,2}(\eta)=\frac{\mathrm{k}}{\omega_{2}-\mathrm{q}_{2, \eta}}\left[\left(\frac{v \omega_{2}}{\mathcal{H}_{2}}\right.\right.$

$\left.-2 q_{2, \eta}^{2}\right) \sinh q_{2, \eta} \ell_{2}-q_{2, \eta}\left(2 \omega_{2}\right.$

$\left.\left.-\frac{v}{u_{2}}\right) \cosh q_{2, \eta} \ell_{2}\right]$.

. $\mathrm{e}^{v \ell_{1} /\left(2 \varkappa_{2}\right)} \cdot \mathrm{e}^{\left(\ell_{1}+\ell_{2}\right) \mathrm{q}_{2, \eta}}$,

$\mathrm{G}_{1, \eta} \equiv \mathrm{G}_{1, \eta}(\mathrm{p}, \mathrm{r})=\overline{\mathrm{F}}_{2, \eta}^{(\mathrm{s})}(\mathrm{p}) \cdot \Phi_{2, \eta}(\mathrm{r})+\overline{\mathrm{F}}_{1, \eta}^{(\mathrm{s})}$

(p). $\Phi_{1, \eta}(\mathrm{r})$,

$\mathrm{G}_{2, \eta} \equiv \mathrm{G}_{2, \eta}\left(\mathrm{B}_{2,3}\right)$

$=\mathrm{k}\left[\frac{v}{2 \mathcal{H}_{2}} \overline{\mathrm{F}}_{2, \eta}^{(\mathrm{s})}(\mathrm{p})+\overline{\mathrm{F}}_{2, \eta}^{(\mathrm{c})}(\mathrm{p})\right] \Phi_{2, \eta}(\mathrm{r})$

$+\left[\frac{v}{2 \varkappa_{1}} \overline{\mathrm{F}}_{1, \eta}^{(\mathrm{s})}(\mathrm{p})+\overline{\mathrm{F}}_{1, \eta}^{(\mathrm{c})}(\mathrm{p})\right] \Phi_{1, \eta}(\mathrm{r})$,

$\Delta_{\eta} \equiv \Delta_{\eta}(\mathrm{p})=\mathrm{C}_{1,1} \cdot \mathrm{C}_{2,2}-\mathrm{C}_{1,2} \cdot \mathrm{C}_{2,1} \quad, \quad \mathrm{k}$

$$
\equiv \mathrm{k}_{2} / \mathrm{k}_{1} \text {, }
$$

$\overline{\mathrm{F}}_{1, \eta}^{(\mathrm{s})}=\frac{1}{\mathcal{u}_{1} \mathrm{q}_{1, \eta}} \int_{0}^{\ell_{1}} \mathrm{e}^{v\left(\ell_{1}-\zeta\right) /\left(2 \varkappa_{1}\right)} \sinh \mathrm{q}_{1, \eta}\left(\ell_{1}\right.$

$$
-\zeta) \cdot \bar{f}_{1, \eta}(\zeta) d \zeta,
$$

$\overline{\mathrm{F}}_{1, \eta}^{(\mathrm{c})}=\frac{1}{\varkappa_{1}} \int_{0}^{\ell_{1}} \mathrm{e}^{v\left(\ell_{1}-\zeta\right) /\left(2 \varkappa_{1}\right)} \cosh \mathrm{q}_{1, \eta}\left(\ell_{1}\right.$

$$
\overline{\mathrm{F}}_{2, \eta}^{(\mathrm{s})}=\frac{1}{\varkappa_{2} \mathrm{q}_{2, \eta}} \int_{\ell_{1}}^{-\zeta) \cdot \overline{\mathrm{f}}_{1, \eta}(\zeta) \mathrm{d} \zeta,} \mathrm{e}^{v\left(\ell_{1}-\zeta\right) /\left(2 \varkappa_{2}\right)} \sinh \mathrm{q}_{2, \eta}\left(\ell_{1}\right.
$$

$\overline{\mathrm{F}}_{2, \eta}^{(\mathrm{c})}=\frac{1}{\varkappa_{2}} \int_{\ell_{1}}^{\ell_{1}+\ell_{2}} \mathrm{e}^{v\left(\ell_{1}-\zeta\right) /\left(2 \varkappa_{2}\right)} \cosh \mathrm{q}_{2, \eta}\left(\ell_{1}\right.$

$$
-\zeta) \cdot \overline{\mathrm{f}}_{2, \eta}(\zeta) \mathrm{d} \zeta
$$

When $0<\mathrm{r}<a$, the constants $\mathrm{A}_{2, \eta}$ are obtained from the relation $\left.\widetilde{T}_{2}\right|_{\mathrm{z}=\ell_{1}}=0$, as 
$\mathrm{A}_{2, \eta}=\overline{\mathrm{F}}_{2, \eta}^{(\mathrm{s})}(\mathrm{p}) / \mathrm{C}_{1,2}(\eta)$.

(28)

The fourth stage: we look for the zeros of the function $\Delta_{\eta}(\mathrm{p})$.

Using these relations for $C_{i, j}(i, j=1,2)$, we write the equation $\Delta_{\eta}(\mathrm{p})=0$ in the form:

$\frac{v}{2}\left(\frac{\mathrm{k}}{\varkappa_{2}}\right.$

$\left.-\frac{1}{x_{1}}\right)\left(\omega_{1} \sinh q_{1, \eta} \ell_{1}\right.$

$\left.-q_{1, \eta} \cosh q_{1, \eta} \ell_{1}\right)\left(\omega_{2} \sinh q_{2, \eta} \ell_{2}\right.$

$\left.+\mathrm{q}_{2, \eta} \cosh \mathrm{q}_{2, \eta} \ell_{2}\right)-$

$-q_{1, \eta}\left(\omega_{1} \cosh q_{1, \eta} \ell_{1}\right.$

$\left.-\mathrm{q}_{1, \eta} \sinh \mathrm{q}_{1, \eta} \ell_{1}\right)\left(\omega_{2} \sinh \mathrm{q}_{2, \eta} \ell_{2}\right.$

$\left.+\mathrm{q}_{2, \eta} \cosh \mathrm{q}_{2, \eta} \ell_{2}\right)-$

$-\mathrm{kq}_{2, \eta}\left(\omega_{1} \sinh \mathrm{q}_{1, \eta} \ell_{1}-\right.$

$\left.\mathrm{q}_{1, \eta} \cosh \mathrm{q}_{1, \eta} \ell_{1}\right)\left(\omega_{2} \cosh \mathrm{q}_{2, \eta} \ell_{2}+\right.$

$\left.\mathrm{q}_{2, \eta} \sinh \mathrm{q}_{2, \eta} \ell_{2}\right)=0$.

if $\varkappa_{1} \lambda_{1, \eta}^{2}+\frac{v^{2}}{4 \varkappa_{1}}$

$>\varkappa_{2} \lambda_{2, \eta}^{2}$

$+\frac{v^{2}}{4 \varkappa_{2}}$, then the equation (29)takes the form :

$\frac{v}{2}\left(\frac{\mathrm{k}}{\mathcal{H}_{2}}\right.$

$\left.-\frac{1}{x_{1}}\right)\left(\omega_{1} \sin \beta_{\eta} \ell_{1}\right.$

$\left.-\beta_{\eta} \cos \beta_{\eta} \ell_{1}\right)\left(\omega_{2} \sin \sqrt{\varkappa \beta_{\eta}^{2}+\gamma_{\eta}^{2}} \ell_{2}\right.$

$\left.+\sqrt{\mathcal{u} \beta_{\eta}^{2}+\gamma_{\eta}^{2}} \cos \sqrt{\mathcal{u} \beta_{\eta}^{2}+\gamma_{\eta}^{2}} \ell_{2}\right)-$

$-\beta_{\eta}\left(\omega_{1} \cos \beta_{\eta} \ell_{1}\right.$

$\left.+\beta_{\eta} \sin \beta_{\eta} \ell_{1}\right)\left(\omega_{2} \sin \sqrt{\mathcal{u} \beta_{\eta}^{2}+\gamma_{\eta}^{2}} \ell_{2}\right.$

$\left.+\sqrt{\varkappa \beta_{\eta}^{2}+\gamma_{\eta}^{2}} \cos \sqrt{\varkappa \beta_{\eta}^{2}+\gamma_{\eta}^{2}} \ell_{2}\right)-\mathrm{k} \sqrt{\varkappa \beta_{\eta}^{2}+\gamma_{\eta}^{2}}$

$\left(\omega_{1} \cos \beta_{\eta} \ell_{1}\right.$

$\left.+\beta_{\eta} \sin \beta_{\eta} \ell_{1}\right)\left(\omega_{2} \cos \sqrt{\mathcal{L} \beta_{\eta}^{2}+\gamma_{\eta}^{2}} \ell_{2}\right.$

$\left.-\sqrt{\varkappa \beta_{\eta}^{2}+\gamma_{\eta}^{2}} \sin \sqrt{\varkappa \beta_{\eta}^{2}+\gamma_{\eta}^{2}} \ell_{2}\right)$

$=0$,

where

$$
\begin{aligned}
& \mathcal{u} \equiv \varkappa_{1} / \varkappa_{2}, \gamma_{\eta}^{2} \\
& \equiv\left(\varkappa_{1} \lambda_{1, \eta}^{2}+\frac{v^{2}}{4 \varkappa_{1}}\right) \\
& -\left(\varkappa_{2} \lambda_{2, \eta}^{2}+\frac{v^{2}}{4 \varkappa_{2}}\right) .
\end{aligned}
$$

The relation (30) is an algebraic equation for $\beta_{\eta}$, we proved that the equation has only real, simple root and infinite numbers

The fifth stage: we apply the inverse Laplace transform with respect to $(t)$ of the two relations (17), (27).

Substituting the relations (23),(27) and (28) into the relations (17),(27)and using Cauchy's theory of residues, on the basis of the results of the fifth stage, we obtain a solution to the problem under study in the form:

$\mathrm{T}_{1}(\mathrm{r}, \mathrm{z}, \mathrm{t})$

$$
\begin{aligned}
& =\mathrm{e}^{-v\left(\ell_{1}-\mathrm{z}\right) /\left(2 \varkappa_{1}\right)} \sum_{\eta=1}^{\infty} \sum_{\mathrm{m}=1}^{\infty} \varphi_{1}\left(\mathrm{z}, \beta_{\eta \mathrm{m}}\right) \\
& \left\{\varphi _ { 2 } ( \ell _ { 2 } , \beta _ { 2 , \eta \mathrm { m } } ) \left[\frac { 1 } { \mathcal { H } _ { 1 } } \beta _ { 2 , \eta \mathrm { m } } \int _ { 0 } ^ { \ell _ { 1 } } \left(\frac { v } { 2 } \left(\frac{\mathrm{k}}{\mathcal{H}_{2}}\right.\right.\right.\right. \\
& \left.-\frac{1}{\varkappa_{1}}\right) \mathrm{R}^{\mathrm{s}}\left(\zeta ; \beta_{\eta \mathrm{m}}\right) \\
& \begin{array}{c}
\left.-\mathrm{R}^{\mathrm{c}}\left(\zeta ; \beta_{\eta \mathrm{m}}\right)\right) \overline{\mathrm{f}}_{1, \eta}(\zeta) \mathrm{d} \zeta \Phi_{1, \eta}(\mathrm{r}) \\
-\frac{\mathrm{k}}{\varkappa_{2}} \beta_{\eta \mathrm{m}} \int_{\ell_{1}}^{\ell_{1}+\ell_{2}} \mathrm{R}_{2}^{\mathrm{c}}\left(\zeta ; \beta_{2, \eta \mathrm{m}}\right) \overline{\mathrm{f}}_{2, \eta}(\zeta) \mathrm{d} \zeta . \Phi_{2, \eta}(\mathrm{r}) \\
\left.-\mathrm{k} \varphi_{2, \mathrm{z}}^{\prime}\left(\ell_{2}, \beta_{2, \eta \mathrm{m}}\right) \mathrm{Q}\left(\mathrm{r}, \beta_{\eta \mathrm{m}}\right)\right\} \mathrm{S}_{\eta \mathrm{m}}(\mathrm{t}),
\end{array}
\end{aligned}
$$

$\mathrm{T}_{2}(\mathrm{r}, \mathrm{z}, \mathrm{t})$

$=\mathrm{e}^{-v\left(\ell_{1}-\mathrm{z}\right) /\left(2 \varkappa_{2}\right)} \sum_{\eta=1}^{\infty} \sum_{\mathrm{m}=1}^{\infty} \varphi_{2}\left(\ell_{1}+\ell_{2}-\mathrm{z}, \beta_{\eta \mathrm{m}}\right)$

$\left\{\varphi_{1}\left(\ell_{1}, \beta_{\eta \mathrm{m}}\right) \frac{1}{\varkappa_{2}}\right.$

$\cdot\left[-\beta_{2, \eta \mathrm{m}} \int_{0}^{\ell_{1}} \mathrm{R}^{\mathrm{c}}\left(\zeta ; \beta_{\eta \mathrm{m}}\right) \overline{\mathrm{f}}_{1, \eta}(\zeta) \mathrm{d} \zeta . \Phi_{1, \eta}(\mathrm{r})+\beta_{\eta \mathrm{m}} \int_{\ell_{1}}^{\ell_{1}+\ell_{2}}\left(\frac{v}{2}\left(\frac{1}{\mathcal{u}_{1}}\right.\right.\right.$

$\left.\left.-\frac{\mathrm{k}}{\mathcal{H}_{2}}\right) \mathrm{R}_{2}^{\mathrm{s}}\left(\zeta ; \beta_{2, \eta \mathrm{m}}\right)-\mathrm{R}_{2}^{\mathrm{c}}\left(\zeta ; \beta_{2, \eta \mathrm{m}}\right)\right)$

.$\left.\overline{\mathrm{f}}_{2, \eta}(\zeta) \mathrm{d} \zeta \cdot \mathrm{K}_{2, \eta}(\mathrm{r})\right]$
$\left.-\varphi_{1, \mathrm{z}}^{\prime}\left(\ell_{1}, \beta_{\eta \mathrm{m}}\right) \mathrm{Q}\left(\mathrm{r}, \beta_{\eta \mathrm{m}}\right)\right\} \cdot \mathrm{S}_{\eta \mathrm{m}}(\mathrm{t})$,

when $a<r<b$,

$\mathrm{T}_{2}(\mathrm{r}, \mathrm{z}, \mathrm{t})$

$=-2 \mathrm{e}^{-v\left(\ell_{1}-\mathrm{z}\right) /\left(2 \varkappa_{2}\right)} \sum_{\eta=1}^{\infty} \sum_{\mathrm{j}=1}^{\infty} \frac{\Phi_{2, \eta}(\mathrm{r}) \varphi_{2}\left(\ell_{1}+\ell_{2}-\mathrm{z}, \beta_{2, \eta \mathrm{j}}\right)}{\mathcal{x}_{2} \beta_{2, \eta \mathrm{j}} \varphi_{2, \beta}^{\prime}\left(\ell_{2}, \beta_{2, \eta \mathrm{j}}\right)}$. 


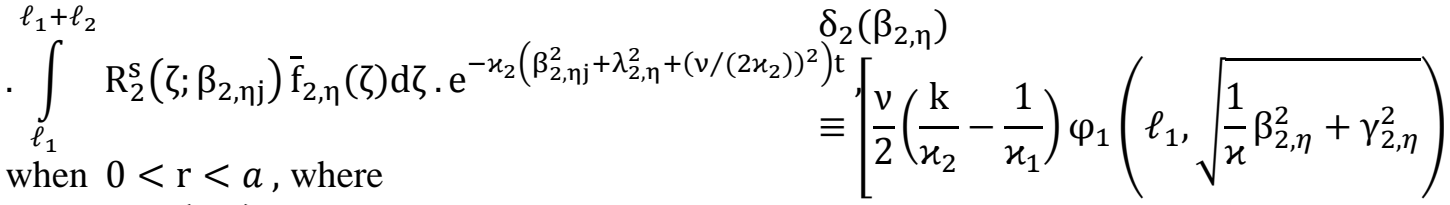

$$
\begin{aligned}
& \varphi_{1}(\mathrm{z}, \beta) \equiv \omega_{1} \sin \beta \mathrm{z}-\beta \cos \beta \mathrm{z} \text {, } \\
& \varphi_{2}(\mathrm{z}, \beta) \equiv \omega_{2} \sin \beta \mathrm{z}+\beta \cos \beta \mathrm{z} \text {, } \\
& \mathrm{R}^{\mathrm{s}}(\mathrm{z} ; \beta) \equiv \mathrm{e}^{v\left(\ell_{1}-\mathrm{z}\right) /\left(2 x_{1}\right)} \sin \beta\left(\ell_{1}-\mathrm{z}\right), \\
& \mathrm{R}_{2}^{\mathrm{s}}(\mathrm{z} ; \beta) \equiv \mathrm{e}^{v\left(\ell_{1}-\mathrm{z}\right) /\left(2 x_{2}\right)} \sin \beta\left(\ell_{1}-\mathrm{z}\right), \\
& \mathrm{R}^{\mathrm{c}}(\mathrm{z} ; \beta) \equiv \mathrm{e}^{v\left(\ell_{1}-\mathrm{z}\right) /\left(2 x_{1}\right)} \beta \cos \beta\left(\ell_{1}-\mathrm{z}\right), \\
& \mathrm{R}_{2}^{\mathrm{c}}(\mathrm{z} ; \beta) \equiv \mathrm{e}^{\mathrm{v}\left(\ell_{1}-\mathrm{z}\right) /\left(2 \mathcal{\varkappa}_{2}\right)} \beta \cos \beta\left(\ell_{1}-\mathrm{z}\right), \\
& \mathrm{Q}\left(\mathrm{r}, \beta_{\eta \mathrm{m}}\right) \\
& \equiv-\frac{1}{\mathcal{H}_{1}} \beta_{2, \eta \mathrm{m}} \int_{\substack{0 \\
\ell_{1}+\ell_{2}}}^{\ell_{1}} \mathrm{R}^{\mathrm{s}}\left(\zeta ; \beta_{\eta \mathrm{m}}\right) \overline{\mathrm{f}}_{1, \eta}(\zeta) \mathrm{d} \zeta . \Phi_{1, \eta}(\mathrm{r}) \\
& -\frac{1}{\mathcal{X}_{2}} \beta_{\eta \mathrm{m}} \int_{\ell_{1}} \mathrm{R}_{2}^{\mathrm{s}}\left(\zeta ; \beta_{2, \eta \mathrm{m}}\right) \overline{\mathrm{f}}_{2, \eta}(\zeta) \mathrm{d} \zeta . \Phi_{2, \eta}(\mathrm{r}), \\
& \text { if }\left(\mathcal{x}_{1} \lambda_{1, \eta}^{2}+\frac{v^{2}}{4 \varkappa_{1}}\right)>\left(\mathcal{x}_{2} \lambda_{2, \eta}^{2}+\frac{v^{2}}{4 \mathcal{x}_{2}}\right) \text {, then } \\
& \beta_{\eta \mathrm{m}} \text { are the positive roots of the equation } \\
& \delta\left(\beta_{\eta}\right) \\
& \equiv\left[\frac{\mathrm{v}}{2}\left(\frac{\mathrm{k}}{\mathcal{u}_{2}}-\frac{1}{\mathcal{H}_{1}}\right) \varphi_{1}\left(\ell_{1}, \beta_{\eta}\right)\right. \\
& \left.-\varphi_{1, \mathrm{z}}^{\prime}\left(\ell_{1}, \beta_{\eta}\right)\right] \varphi_{2}\left(\ell_{2}, \sqrt{\varkappa \beta_{\eta}^{2}+\gamma_{\eta}^{2}}\right) \\
& -\mathrm{k} \cdot \varphi_{1}\left(\ell_{1}, \beta_{\eta}\right) \varphi_{2, \mathrm{z}}^{\prime}\left(\ell_{2}, \sqrt{\mathcal{u} \beta_{\eta}^{2}+\gamma_{\eta}^{2}}\right)=0, \\
& \begin{array}{l}
\left.-\varphi_{1, \mathrm{z}}^{\prime}\left(\ell_{1}, \sqrt{\frac{1}{\mathcal{x}} \beta_{2, \eta}^{2}+\gamma_{2, \eta}^{2}}\right)\right] \varphi_{2}\left(\ell_{2}, \beta_{2, \eta}\right) \\
- \text { k. } \varphi_{1}\left(\ell_{1}, \sqrt{\frac{1}{x} \beta_{2, \eta}^{2}+\gamma_{2, \eta}^{2}}\right) \varphi_{2, \mathrm{z}}^{\prime}\left(\ell_{2}, \beta_{2, \eta}\right)
\end{array} \\
& =0 \text {, } \\
& \beta_{\eta \mathrm{m}} \equiv \sqrt{\frac{1}{\mathcal{x}} \beta_{2, \eta}^{2}+\gamma_{2, \eta}^{2}}, \\
& \gamma_{2, \eta}^{2} \\
& \equiv \frac{1}{\varkappa_{1}}\left[\left(\varkappa_{2} \lambda_{2, \eta}^{2}+\frac{v^{2}}{4 \varkappa_{2}}\right)\right. \\
& \left.-\left(\varkappa_{1} \lambda_{1, \eta}^{2}+\frac{v^{2}}{4 \varkappa_{1}}\right)\right] \text {, } \\
& S_{\eta \mathrm{m}}(\mathrm{t})=\frac{2}{\beta_{\eta \mathrm{m}} \beta_{2, \eta \mathrm{m}} \delta_{2}^{\prime}\left(\beta_{2, \eta \mathrm{m}}\right)} \exp \left\{-\varkappa_{2}\left(\beta_{2, \eta \mathrm{m}}^{2}\right.\right. \\
& \left.\left.+\lambda_{2, \eta}^{2}+\left(v /\left(2 \varkappa_{2}\right)\right)^{2}\right) t\right\}
\end{aligned}
$$$$
\beta_{2, \eta \mathrm{m}} \equiv \sqrt{\varkappa \beta_{\eta}^{2}+\gamma_{\eta}^{2}}
$$$$
\gamma_{\eta}^{2}
$$$$
\equiv \frac{1}{\varkappa_{2}}\left[\left(\varkappa_{1} \lambda_{1, \eta}^{2}+\frac{v^{2}}{4 \varkappa_{1}}\right)\right.
$$$$
\left.-\left(x_{2} \lambda_{2, \eta}^{2}+\frac{v^{2}}{4 \varkappa_{2}}\right)\right]
$$$$
\mathrm{S}_{\eta \mathrm{m}}(\mathrm{t})=\frac{2}{\beta_{\eta \mathrm{m}} \beta_{2, \eta \mathrm{m}} \delta^{\prime}\left(\beta_{\eta \mathrm{m}}\right)} \exp \left\{-\varkappa_{1}\left(\beta_{\eta \mathrm{m}}^{2}\right.\right.
$$$$
\left.\left.+\lambda_{1, \eta}^{2}+\left(v /\left(2 \varkappa_{1}\right)\right)^{2}\right) \mathrm{t}\right\} \text {, }
$$$$
\text { if }\left(\varkappa_{2} \lambda_{2, \eta}^{2}+\frac{v^{2}}{4 \varkappa_{2}}\right)>\left(\varkappa_{1} \lambda_{1, \eta}^{2}+\frac{v^{2}}{4 \varkappa_{1}}\right) \text {, then }
$$$$
\beta_{2, \eta \mathrm{m}} \text { are the positive roots of the equation }
$$

\section{Conclusion}

In this paper, the spread heat in a body which in two cylinders is studied. The solution behavior of the boundary problem is given as an infinite series using two sequences of integral transformation forms, Bessel function theory and the conditions of contact between the two cylinders. Hence we get a transcendental equation and using the theorem of type Dixon, we proved that this equation has infinitely, simple and real roots. The behavior of the eigenvalues spectral problem is determined. The obtained Eigen functions are the kernel of integral transformations of first kind Bessel function. This problem has numerous engineering applications, such as, the administration motion of bodies which consists of two cylinders or more. Also, machining, welding, grinding, internal combustion engines, as well as in the factories for the production of military weapons, are all others practical examples. 


\section{Acknowledgements}

The authors are very much grateful to Professor Ahmed M. K. Tarabia and Dr. R. Tantawy members of mathematics department at Damietta University, Egypt for their valuable comments to bring the paper in the present form.

\section{References}

Carslaw, H.S. and Jaeger, J.C., Conduction of Heat in Solids, Clarendon Press, Oxfor, 1984.

Gasimov , G.R. , Rzayev , E.A. , Aliev , A.M. , Investigation of a solution problem of heat conduction in the moving bounded hollow cylinder Proceedings of the Institute of Applied Mathematics, Baku State University, V.2, No.1, 2013.

hollow cylinder using the program Maple11,The Second Conference on Mathematical Sciences,
Kholodovskii , S.Y. , Solving Boundary Value Problems in Cylinders with Multilayer Film inclusion, J, Scientific Notes of Zabaikalskii State University, N.3, 2012.

Kuznetsova, Y.A. , Temperature filed of finite hollow radial and inhomogeneous moving cylinder, J. New of Tula State University, Series of differential equations and applied problems, N.1, 2005 .

Lotarev, V.Y., Temperature filed of finite continuous moving cylinder, J. New of Tula State

M.Necati( O) "zi_s ik, Heat Conduction, New York, 1993.

Shahout ,A.M., Gasimov , G.R. , Rzayev , E.A. , Solution problem of heat conduction in the circular University, V.5, N.2, 1999.

Watson, G.N., A Treatise on the Theory of Bessel Function, Cambridge, ed.2, 1944.

Zarqa Private University, Jordan, October 22-23, 2008

\footnotetext{
الملخص العربي

عنوان البحث: معادلة التوصيل الحرارى فى وسط غير متجانس للأجسام الصلبة المركبة المتحركة

قربان جاسيموف1، زهرة على أبوسطاش2

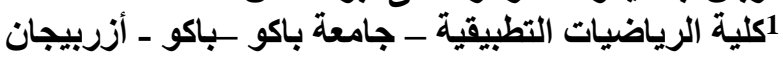

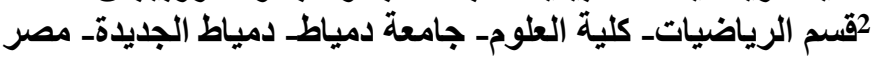

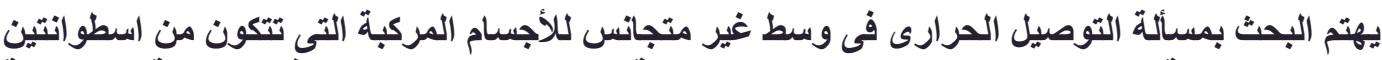

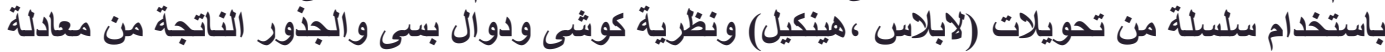

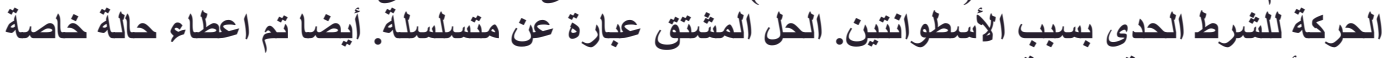

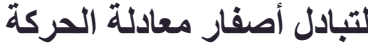

